Paper

\title{
Distributed power saving scheme in consideration of QoS requirement for IEEE 802.11e network
}

\author{
Nobuyoshi Komuro ${ }^{1 \text { a) }}$ \\ ${ }^{1}$ Graduate School of Advanced Integration Science, Chiba University \\ 1-33 Yayoi-cho, Inage-ku, Chiba 263-8522, Japan \\ a) kmr@faculty.chiba-u.jp
}

Received July 18, 2014; Revised November 15, 2014; Published April 1, 2015

\begin{abstract}
This paper proposes a scheme for the distributed power saving mechanism of IEEE 802.11e networks in consideration of the Quality of Service requirement. In the proposed protocol, each station enters into sleep-mode and active-mode periodically according to the appropriate trigger interval. For obtaining the appropriate trigger interval, this paper presents the objective function of power consumption in consideration of the acceptable delay required by each access category. Each station optimizes its trigger interval according to the objective function. Simulation results show the effectiveness of the proposed scheme.
\end{abstract}

Key Words: power saving mechanism, Quality of Service, optimization

\section{Introduction}

The increasing popularity of wireless Internet access in mobile device such as PDA or smart phones increases the incorporation of the widely spread distributed wireless network technology. These devices pose two challenges: One is to satisfy Quality of Service (QoS) requirements such as delay and throughput, and the other is to save consumed power. Many protocols were proposed for overcoming these challenges [2-14]. IEEE 802.11e [1] was standardized as the MAC protocol to support the high-priority flows. IEEE 802.11e Enhance Distributed Channel Access (EDCA) is defined in IEEE 802.11e (extended IEEE 802.11 Distributed Coordination Function). Specific stations (flows) take high-priority and preferentially use wireless resources. Unscheduled Automatic Power Save Delivery (U-APSD), which is defined as a distributed power saving mechanisms in IEEE 802.11e, addresses the above challenges. In U-APSD, each station enters into sleep-mode and active-mode periodically according to the trigger interval. The delay introduced by U-APSD can be reduced compared with the basic Power Save Mode (PSM) in IEEE 802.11 because a trigger frame can be sent at any point in time. However, it is difficult to decide a trigger interval because QoS of data-frame depends on the algorithm which decides when to send trigger frame for receiving the buffered frames. In addition, there is a trade-off between QoS and the power consumption in U-APSD.

The schemes in [3-5] adaptively configured distributed power saving mechanisms in order to meet the QoS required by the applications. In those schemes, each station decides the trigger interval 
based on the data-frame arrival interval without using any control messages from the Access Point (AP). Since it is an extension of U-APSD, it is possible to keep QoS with saving power consumption. However, since those schemes do not consider the acceptable delay of each access category, highpriority data-frames results in extra delay. Extra delay is one of the serious problems for high-priority data-frame.

The power saving schemes for IEEE 802.11, which decides power management mode of each station according to an optimal sequence such that the acceptable delay is guaranteed with minimum power consumption, were proposed in $[13,14]$. The scheme in [13] obtained the objective function of power consumption in consideration of the acceptable delay and the trigger interval, which minimizes the objective function. However, since the scheme in [13] is based on IEEE 802.11 PSM, it is difficult to decide the trigger interval in order to guarantee QoS required by each access category. In addition, since behavior of each station is controlled by the AP, it is difficult to extend to distributed networks.

This paper, which was previously presented in part at KJCCS 2013 [15], proposes a scheme for the distributed power saving mechanism of IEEE 802.11e networks in consideration of the QoS requirement. In the proposed protocol, each station enters into sleep-mode and active-mode periodically according to the appropriate trigger interval. For obtaining the appropriate trigger interval, this paper presents the objective function of power consumption in consideration of the acceptable delay required by each access category. Each station optimizes its trigger interval according to the objective function. The delay and the power consumption of the proposed scheme are evaluated by simulations.

\section{Related Works}

\subsection{U-APSD}

Unscheduled Automatic Power Save Delivery (U-APSD) is the distributed APSD method, which is defined in 802.11e for improving the QoS provided to EDCA-compliant stations. The basic idea of the U-APSD is to use a specific interval, which is called Unscheduled Service Period (U-SP), for an AP to deliver data-frames to a station and to use trigger sending for requesting buffered frames in the AP. Figure 1 shows the example scenario of U-APSD. The station using U-APSD can start U-SP with sending trigger frame. After the AP responds with ACK, a U-SP starts and the AP begins to deliver data-frames, which is destined for the EDCA-compliant station. During the U-SP, the AP

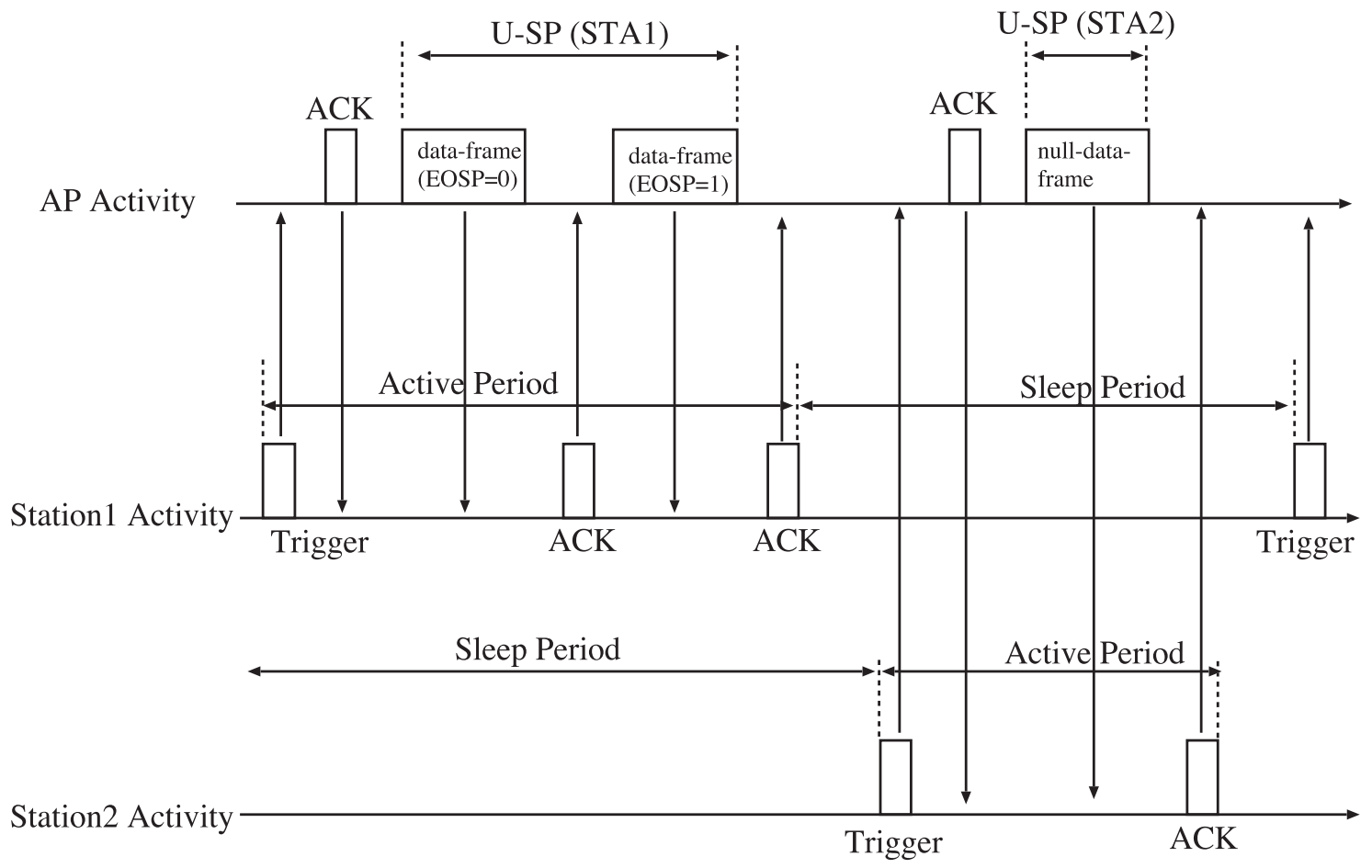

Fig. 1. Example scenario of U-APSD. 


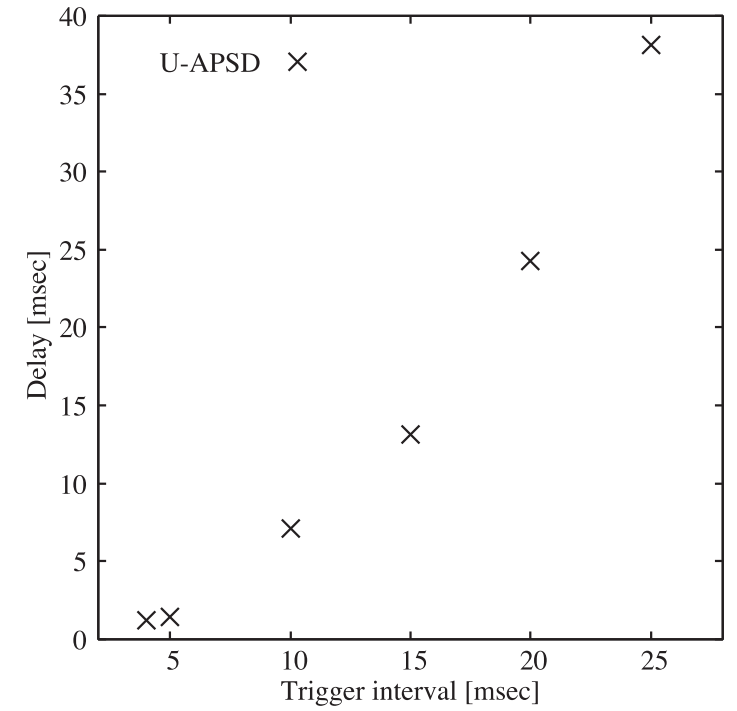

(a) Delay

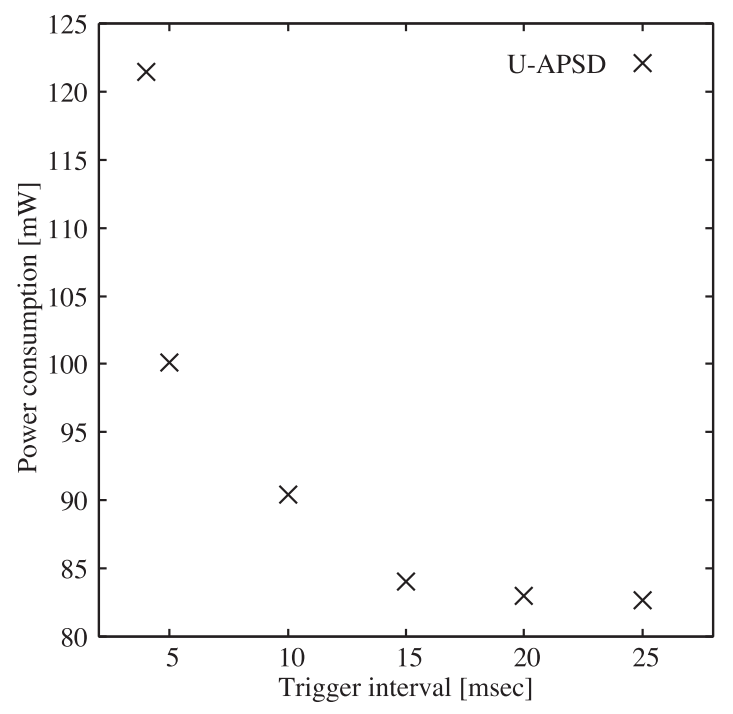

(b) Power consumption

Fig. 2. Delay and power consumption of U-APSD as a function of trigger interval.

delivers at least one data-frame to the station. If the AP receives a trigger frame from the station and has no buffered frame destined for the station, the AP sends a null-data frame to the station because the AP need to send at least one frame to the station. At the end of a U-SP, the AP informs the station about the end of the U-SP using End of Service Period (EOSP) field set to 1 in QoS control field in data-frame. When receiving null-data frame or data-frame with EOSP set to 1, the station enters into sleep mode and the AP cannot deliver further data-frames until a new U-SP starts. If there are more buffered data-frames destined for the station, the AP sets EOSP field in frame control field to 0 and informs the station that more frames destined to the station are buffered. The delay introduced by U-APSD can be reduced compared with the basic power save mode because a trigger can be generated at any point in time.

\subsection{Problem of U-APSD}

It is difficult to decide a trigger interval because QoS of data-frame depends on the algorithm which decides when to send trigger frame for receiving the buffered frames. Figure 2 shows the delay and power consumption of a U-APSD-compliant station. It is seen from Figs. 2 that there is a trade-off between the delay and the power consumption in U-APSD. It is also seen from Fig. 2 that the delay significantly increases while the power saving becomes less effective when the trigger interval is long. Many studied proposed the way to decide trigger interval [3-8].

\subsection{Enhanced U-APSD}

The schemes in [3-5] adaptively configured distributed power saving mechanisms in order to meet the QoS required by the applications. If a station sends trigger frames at the same rate that data-frames arrive at the AP, each trigger frame will result in a single frame delivered by the AP. If a station sends trigger frames at a rate that is above the rate of arrivals at the AP, each trigger frame will result in either one or no frames delivered by the AP. If a station sends trigger frames at a rate below the rate of arrivals at the AP, each trigger frame will result in one or more frames delivered by the AP. According to the above observations, the enhanced U-APSD, which was proposed in [4], decides the trigger interval according to

$$
T_{t r g}(n+1)=T_{t r g}(n)-\gamma \frac{\partial}{\partial T_{t r g}}\left(T_{t r g}-\overline{T_{D L}}\right)^{2},
$$

where $T_{t r g}(n)$ represents the current value of trigger interval, $\overline{T_{D L}}$ is the average data-frame arrival interval, and $\gamma<1$. By using the obtained $T_{t r g}$, it is possible to save power consumption with keeping 
QoS. However, since the enhanced U-APSD does not consider the acceptable delay required by each access category, high-priority flows result in extra delay.

\subsection{Smart PSM}

The power saving schemes for IEEE 802.11, which decide the power management mode of each station according to an optimal sequence such that the acceptable delay performance is guaranteed with minimum power consumption, were proposed in [13, 14]. In [13,14], the AP keeps tracks of action sequences, which include trigger interval, active period, and sleep period, of each station. In [13], an acceptable delay is translated into a penalty function. The objective function of power consumption in consideration of the penalty function was derived. The AP calculates action sequences according to the objective function. The AP informs each station about calculated action sequences. Each station can save power consumption with keeping the acceptable delay by following the action sequences. However, it is difficult for the scheme in [13] difficult to decide the appropriate trigger interval for guaranteeing QoS required by each access category because the required QoS and the required powersaving-effect differs by access category. Also, since the behavior of each station is controlled by the $\mathrm{AP}$, it is difficult to extend to distributed networks.

\section{System Model}

The basic idea of the proposed scheme is to derive trigger interval in U-APSD in consideration of the power consumption and the acceptable delay.

Figure 3 shows the flowchart of the proposed scheme. At first, each station initiates Sleep Timer (ST). A station, which is in the sleep mode, wakes up when ST becomes 0. After waking up, the station sends a trigger frame to the AP for requesting each of buffered data-frames and sets Waiting Timer (WT) for ACK reception from the AP (ACK-WT). If the station receives an ACK frame from the AP within ACK-WT, it sets WT for data-frame-reception (Data-WT). If the station does not receive an ACK frame within ACK-WT, it re-sends a trigger frame to the AP. If the number of re-sendings exceeds retry limit (RL), the station sets ST and enters into sleep-mode.

If the station does not receive a data-frame within Data-WT, the station prepares for triggerframe-sending. If the station receives a frame during Data-WT, it checks whether the received frame is null-data frame or not. If the received frame is null-data frame, the station sends an ACK-frame to the AP. After sending the ACK-frame, the station sets ST and enters into sleep-mode. If the received frame is not null-data frame, the station records the access category, reception time, and reception number of the frame. Based on the recorded reception time and reception number, the station estimates the data-frame arrival interval. The arrival interval of $i$-th data-frame is estimated by

$$
T_{D L}(i)=\alpha[\text { reception_time }(i)-\text { reception_time }(i-1)]+(1-\alpha) T_{D L}(i-1),
$$

where $\alpha(<1.0)$ is a smoothing factor.

After recording the reception time, the station checks the value of EOSP. If the value of EOSP is 0, the station sends an ACK frame to the AP and sets Data-WT and waits for the next data-frame. If the value of EOSP is 1, the station sends an ACK frame to the AP and determines the trigger interval based on the estimated data-frame arrival interval and the access category. After determining the trigger interval, the station sets ST and enters into sleep-mode. The way to derive trigger interval is explained in the next section.

\section{Objective function}

This section presents the objective function for deriving the optimum trigger interval in consideration of the power consumption and the delay of each access category in IEEE 802.11e network. As well as the objective function in [13], the proposed objective function consists of the function of power consumption and the penalty function of delay. 


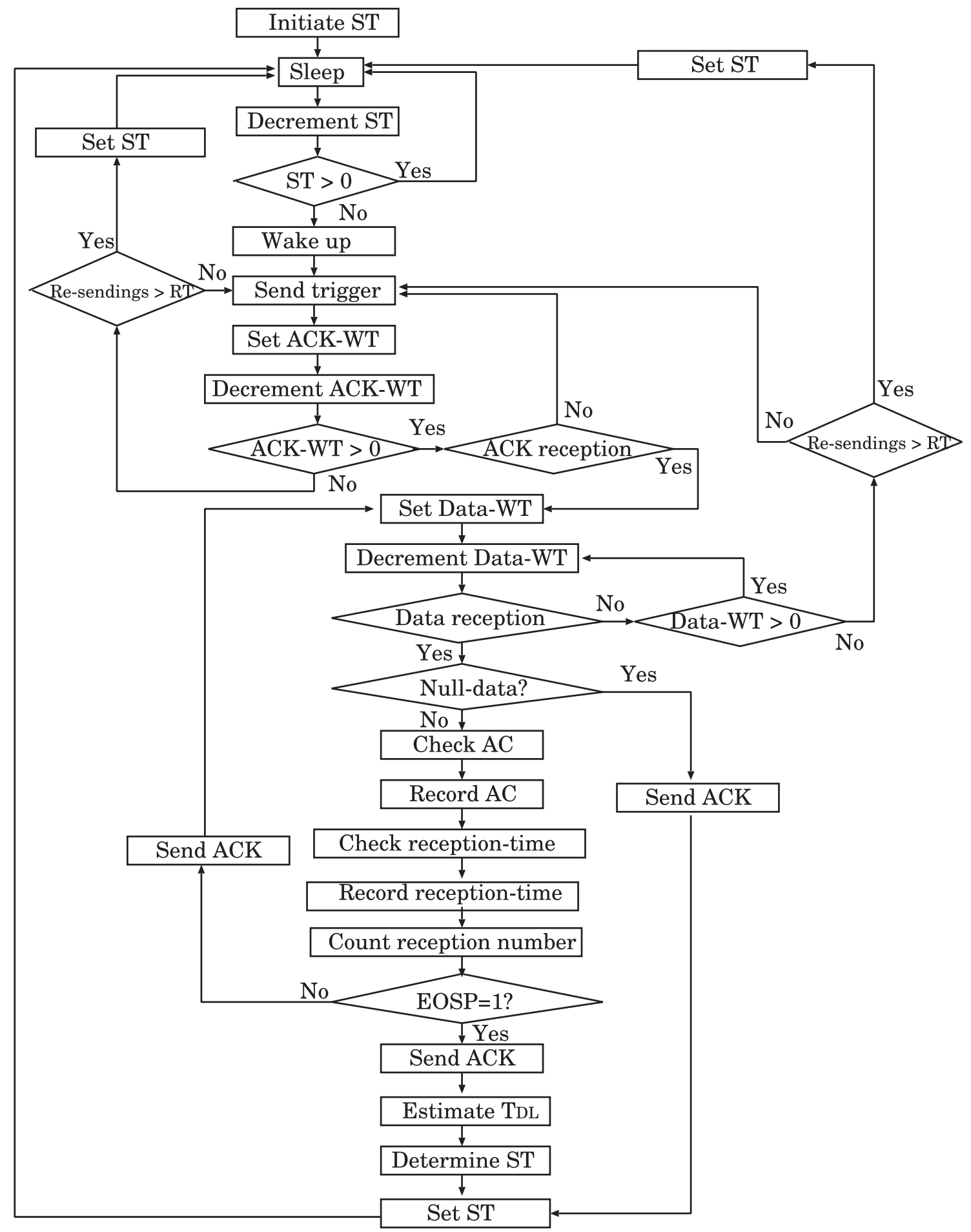

Fig. 3. Flowchart of the proposed scheme.

\subsection{Function of power consumption}

Let $T_{t r g}$ and $T_{D L}$ be trigger interval and the data-frame arrival interval, respectively. The number of data-frames, which a station receives during one U-SP, is

$$
n=\frac{T_{t r g}}{T_{D L}} .
$$

The active period for one station consists of idle time, trigger-sending time, ACK reception time, dataframe reception time, and ACK sending time. The sending period, the reception period, and the idle period are $T_{\text {send }}=T R I G G E R+A C K, T_{\text {receive }}=n(F R A M E+A C K)$, and $T_{\text {idle }}=(n+1)(D I F S+$ $2 S I F S)$, respectively. DIFS, SIFS, TRIGGER, ACK, and FRAME, are distributed inter-frame 
space duration, shot inter-frame space duration, trigger frame-length, ACK frame length, and dataframe-length, respectively. The active period and the sleep period are $T_{\text {active }}=T_{\text {send }}+T_{\text {receive }}+T_{\text {idle }}$ and $T_{\text {sleep }}=T_{\text {trg }}-T_{\text {active }}$, respectively. The power consumption for one cycle as a function of $T_{\text {trg }}$ is

$$
\operatorname{Pw}\left(T_{\text {trg }}\right)=\frac{T_{\text {send }} P_{\text {send }}+T_{\text {receive }} P_{\text {receive }}+T_{\text {idle }} P_{\text {idle }}+\left(T_{\text {trg }}-T_{\text {active }}\right) P_{\text {sleep }}}{T_{\text {trg }}},
$$

where $P_{\text {send }}$ is the power consumption of sending-mode, $P_{\text {receive }}$ is that of reception mode, $P_{i d l e}$ is that of idle mode, and $P_{\text {sleep }}$ is that for sleep mode.

\subsection{Penalty function for delay}

If the number of buffered data-frames in the AP during $T_{t r g}$ destined for a station is more than 1 , the $\mathrm{AP}$ has at least one data-frame in its buffer, which causes the extra delay. The number of buffered data-frames in the AP destined for a station is

$$
N=\frac{T_{t r g}}{2 T_{D L}\left(\frac{1}{1+\frac{1}{T_{\text {frame }^{T} D L}}}\right)},
$$

where $T_{\text {frame }}=S I F S+D I F S+F R A M E$. For reducing the effect of the extra delay, $N$ should be less than 1 . On the other hand, for saving the power consumption, $N$ should be equal to or more than one. Let $p_{A C}$ be a factor for delay bound of each access category. The penalty function for delay, which is no unit function, is

$$
D\left(T_{\text {trg }}\right)=1+\left(\frac{T_{t r g}}{2 p_{A C} T_{D L}\left(1+\frac{1}{T_{\text {frame }} T_{D L}}\right)}\right)^{z} .
$$

$z$ reflects the extent to which the station is willing to tolerate the extra delay. The acceptable delay becomes harder as $z$ increases. The extra delay is bounded by $\frac{T_{t r g}}{2 p_{A C} T_{D L}\left(1+\frac{1}{T_{\text {frame }} T_{D L}}\right)}$. The delay bound is decided by slowdown factor $p_{A C}$. Figure 4 shows the penalty function $D\left(T_{t r g}\right)$ for different $p_{A C}$, and that for different $z$. It is seen from Fig. 4 that $p_{A C}$ and $z$ lead to the extra delay tolerance level. The qualitative description of the acceptable delay and corresponding parameters $\left(p_{A C}\right.$ and $\left.z\right)$ are presented in Table I.

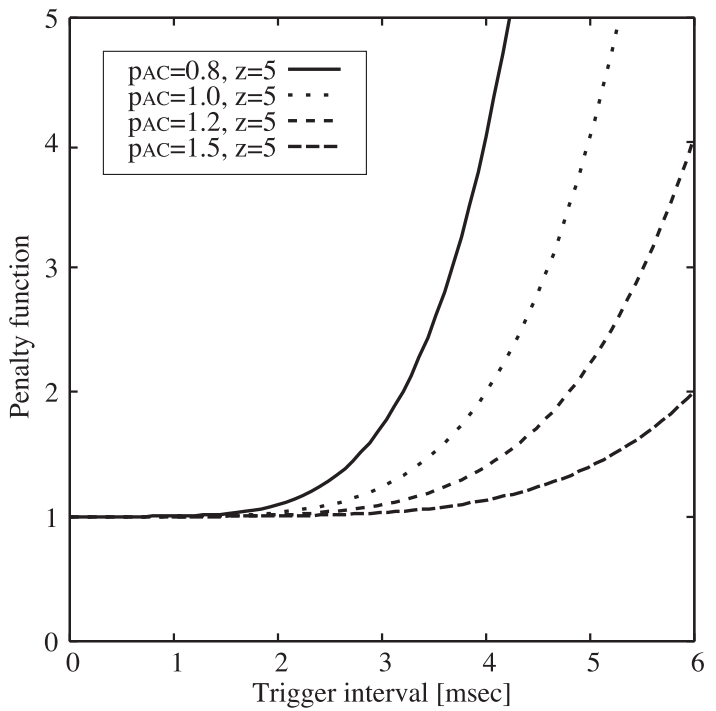

(a) Penalty function for different $p_{A C}$

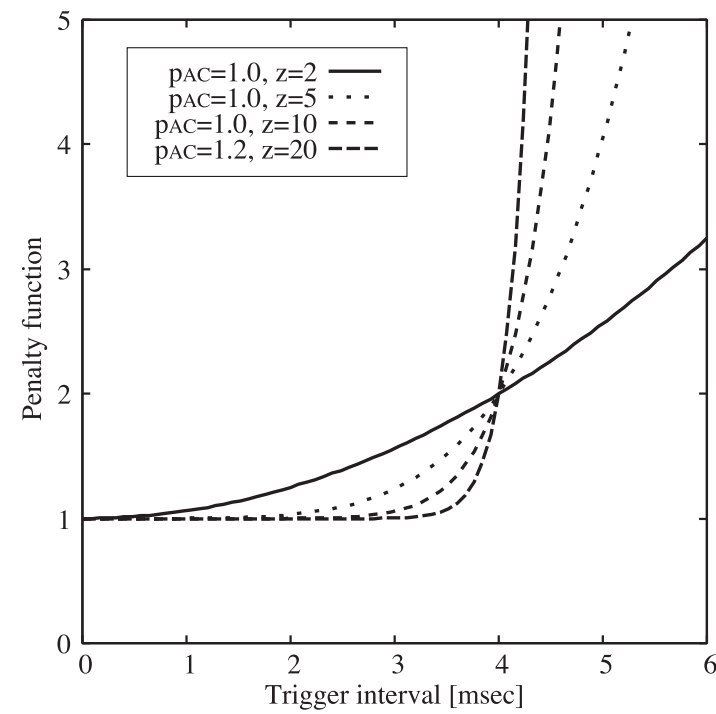

(b) Penalty function for different $z$

Fig. 4. Penalty function as a function of trigger interval. 
Table I. Acceptable delay and corresponding parameters.

\begin{tabular}{c|c|c|c}
\hline \hline Priority $(\mathrm{AC})$ & Extra delay tolerance level & $z$ & $p_{A C}$ \\
\hline \hline high & low & high & low \\
\hline medium & medium & medium & medium \\
\hline low & high & low & high \\
\hline \hline
\end{tabular}

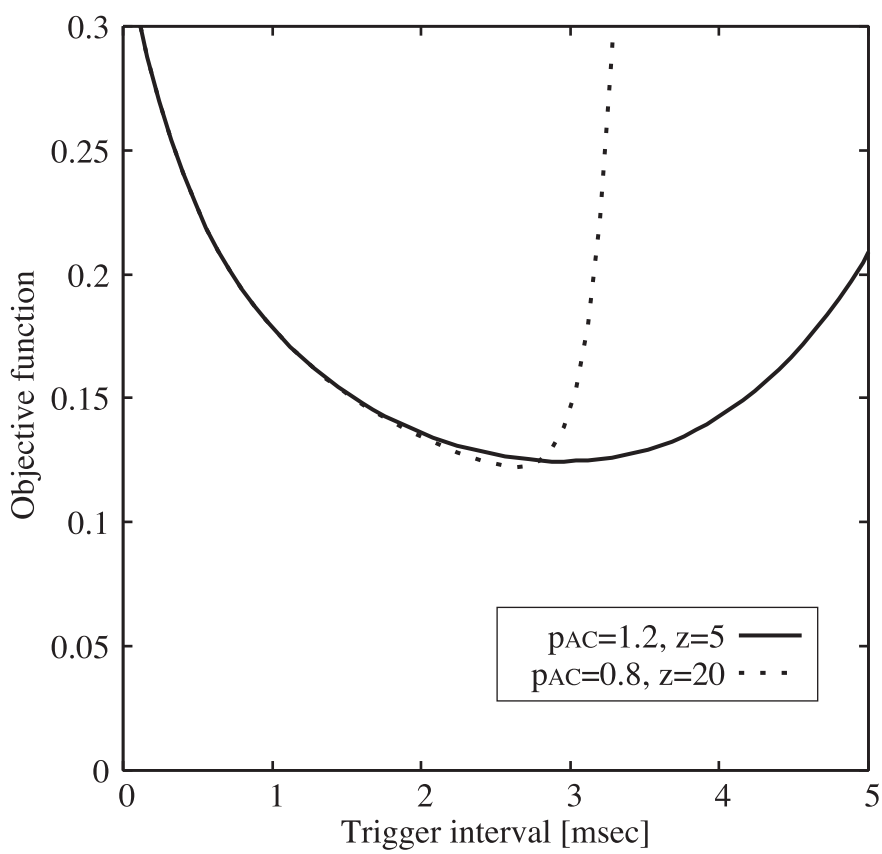

Fig. 5. Example of the objective function for different $p$.

Table II. Parameter for the example of objective function.

\begin{tabular}{c|c}
\hline \hline Parameter & Value \\
\hline \hline ACK & $18[\mu \mathrm{s}]$ \\
\hline DIFS & $34[\mu \mathrm{s}]$ \\
\hline SIFS & $16[\mu \mathrm{s}]$ \\
\hline FRAME & $682[\mu \mathrm{s}]$ \\
\hline TEIGGER & $18[\mu \mathrm{s}]$ \\
\hline$P_{\text {send }}$ & $660[\mathrm{~mW}]$ \\
\hline$P_{\text {receive }}$ & $395[\mathrm{~mW}]$ \\
\hline$P_{\text {idle }}$ & $35[\mathrm{~mW}]$ \\
\hline$T_{D L}$ & $4[\mathrm{~ms}]$ \\
\hline$\left(p_{A C}, z\right)$ & $(1.2,5),(0.8,20)$ \\
\hline
\end{tabular}

\subsection{Objective function}

Based on Eqs. (3) and (5), the objective function $f\left(T_{t r g}\right)$ is

$$
f\left(T_{t r g}\right)=P w\left(T_{t r g}\right) \cdot D\left(T_{t r g}\right) .
$$

The optimized trigger timing is obtained by

$$
T_{t r g}^{*}=\underset{T_{t r g} \in \mathbf{R}}{\arg \min } f\left(T_{t r g}\right)
$$

Figure 5 shows an example of the objective function. Parameters, which are used in the example, are shown in Table II. The value of the objective function decreases as the trigger interval increases because of the effect of power saving. On the other hand, the value of the objective function increases after the trigger interval exceeds delay bound because of the effect of the penalty function. It is seen 
from Fig. 5 that the minimum value of $f\left(T_{t r g}\right)$ and $T_{t r g}^{*}$ depend on $p_{A C}$ and $z$. The proposed scheme can save power in consideration of QoS required by each access category by using corresponding parameters to the acceptable delay.

\section{Evaluation}

The proposed scheme is evaluated by NS-2 simulator. Simulation parameters are shown in Table III. Simulation topology is shown in Fig. 6. In the simulation, there are four stations. Station 1 sends high-priority data (AC_VI) to Station 2 via the AP. Station 3 sends low priority data (AC_BE) to Station 4 via the AP. Data-frames are generated according to the Poisson arrival with arrival rate $1 / T_{D L}$. Power consumption of sending is $660[\mathrm{~mW}]$, that of reception is $395[\mathrm{~mW}]$, that of idle state is $35[\mathrm{~mW}]$, and that of sleep state is $1[\mathrm{~mW}]$. The buffer size of the AP is 100 [packets]. The minimum contention window size $C W_{\min }$ used for $\mathrm{AC} \_\mathrm{VI}$ is 3 , and that for AC_BE is 15 . The

Table III. Simulation parameter.

\begin{tabular}{c|c}
\hline \hline Parameter & Value \\
\hline \hline Data Rate & $6[\mathrm{Mbps}]$ \\
\hline Basic Rate & $6[\mathrm{Mbps}]$ \\
\hline Data Payload & $512[\mathrm{bytes}]$ \\
\hline ACK & $14[\mathrm{bytes}]$ \\
\hline DIFS & $34[\mu \mathrm{s}]$ \\
\hline SIFS & $16[\mu \mathrm{s}]$ \\
\hline TRIGGER & $18[\mu \mathrm{s}]$ \\
\hline Retry Limit & 7 \\
\hline$C W_{\text {min }}($ AC_VI $)$ & 3 \\
$C W_{\text {max }}($ AC_VI $)$ & 7 \\
\hline$C W_{\text {min }}\left(\mathrm{AC} \_B E\right)$ & 15 \\
$C W_{\text {max }}(\mathrm{AC}$ BEE $)$ & 1023 \\
\hline$P_{\text {send }}$ & $660[\mathrm{~mW}]$ \\
\hline$P_{\text {receive }}$ & $395[\mathrm{~mW}]$ \\
\hline$P_{\text {idle }}$ & $35[\mathrm{~mW}]$ \\
\hline$P_{\text {sleep }}$ & $1[\mathrm{~mW}]$ \\
\hline$T_{D L}$ & $4[\mathrm{~ms}]$ \\
\hline$z\left(A C_{-} V I\right)$ & 20 \\
$z\left(A C_{-} B E\right)$ & 5 \\
\hline$p_{A C_{-} V I}$ & 0.8 \\
$p_{A C_{-} B E}$ & 1.2 \\
\hline Simulation time & $100[\mathrm{~s}]$ \\
\hline \hline
\end{tabular}

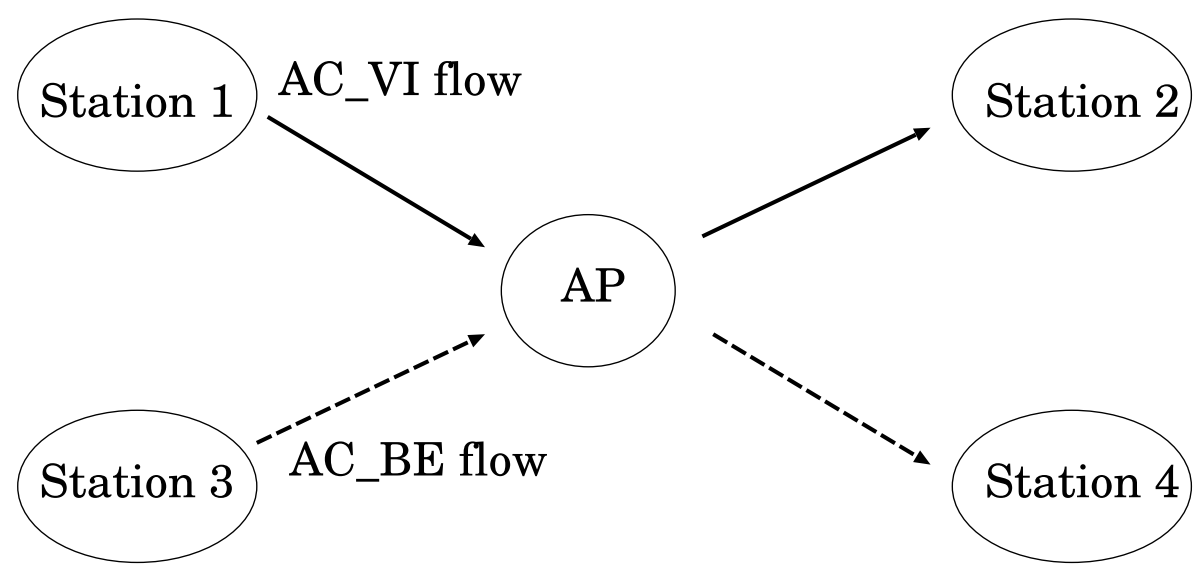

Fig. 6. Simulation topology. 
Table IV. Average delay of each scheme.

\begin{tabular}{c|c|c}
\hline \hline Protocol & AC_VI & AC_BE \\
\hline \hline Proposed & $1.35[\mathrm{~ms}]$ & $2.44[\mathrm{~ms}]$ \\
\hline Conventional & $1.58[\mathrm{~ms}]$ & $2.31[\mathrm{~ms}]$ \\
\hline \hline
\end{tabular}

Table V. Delay-variance of each scheme.

\begin{tabular}{c|c|c}
\hline \hline Protocol & AC_VI & AC_BE \\
\hline \hline Proposed & 0.85 & 8.09 \\
\hline Conventional & 1.86 & 7.49 \\
\hline \hline
\end{tabular}

Table VI. Power consumption of each scheme.

\begin{tabular}{c|c|c}
\hline \hline Protocol & AC_VI & AC_BE \\
\hline \hline Proposed & $172.7[\mathrm{~mW}]$ & $146.7[\mathrm{~mW}]$ \\
\hline Conventional & $155.8[\mathrm{~mW}]$ & $153.5[\mathrm{~mW}]$ \\
\hline \hline
\end{tabular}

maximum contention window size $C W_{\max }$ used for $\mathrm{AC}_{-} \mathrm{VI}$ is 7 , and that for $\mathrm{AC} \_\mathrm{BE}$ is 1023 . The delays and the power consumptions of following four flows are evaluated: AC_VI-flow of the proposed scheme, AC_BE-flow of the proposed scheme, AC_VI-flow of the scheme in [4] (enhanced U-APSD), and AC_BE-flow of the enhanced U-APSD. The delay is defined as the average time between dataframe-sending time and data-frame-receiving time. The power consumption is defined as the average consumed power in the simulation time.

Tables IV and V show the average delay and the delay-variance of each scheme, respectively. It is seen from Tables IV and V that the AC_VI-flow-delay of the proposed scheme is better than that of the U-APSD. This is because the station, which receives high-priority data, emphasizes the low delay rather than the low power consumption in the proposed scheme. On the other hand, the AC_BE-flow-delay of the proposed scheme is worse than that of the U-APSD.

Table VI shows the power consumption of the proposed scheme and that of the enhanced U-APSD. Since the station, which receives AC_VI-flow, emphasizes the low delay rather than the effect of power-consumption-reduction in the proposed scheme, the power consumption of the station with AC_VI-flow is higher than that with AC_BE-flow. In the enhanced U-APSD, the power consumption of the station with AC_VI-flow is almost the same as that with AC_BE-flow because the trigger interval of each station is almost the same. It is seen from Tables IV and VI that the decrease-rate in delay and the increase-rate in power consumption of the proposed scheme are almost the same. This is because the effect on delay becomes large especially when the data-frame arrival is short. In order to avoid the extra delay, the stations, which receives high-priority data, sacrifice the effect on power saving.

Figure 7 shows the delay of the proposed scheme and that of the enhanced U-APSD as a function of the data-frame arrival interval. The delay of AC_VI-flow in the proposed scheme is lower than those of the other flows at any data-frame arrival interval. This is because the trigger interval for AC_VI-flow is set to short value in order not to increase the number of buffered data-frames in the AP. On the other hand, the delay of the AC_BE-flow in the proposed scheme is almost the same as that of the enhanced U-APSD. Since the proposed scheme considers the acceptable delay of each AC, the AC_VI-flow-delay of the proposed scheme is lower than that of the enhanced U-APSD.

Figure 8 shows the power consumption of the proposed scheme and that of the enhanced U-APSD as a function of data-frame arrival interval. The power consumption of the station with AC_VIflow of the proposed scheme is the highest of all stations. Especially when $T_{D L}$ is short, the power consumption of the station with AC_VI-flow of the proposed scheme is higher than those of the other stations. When $T_{D L}$ is short, the station frequently sends trigger frame for requesting high-priority data-frame, which causes the high power consumption. On the other hand, power consumption of the station with AC_VI-flow of the proposed scheme is almost the same as those of the other stations when 


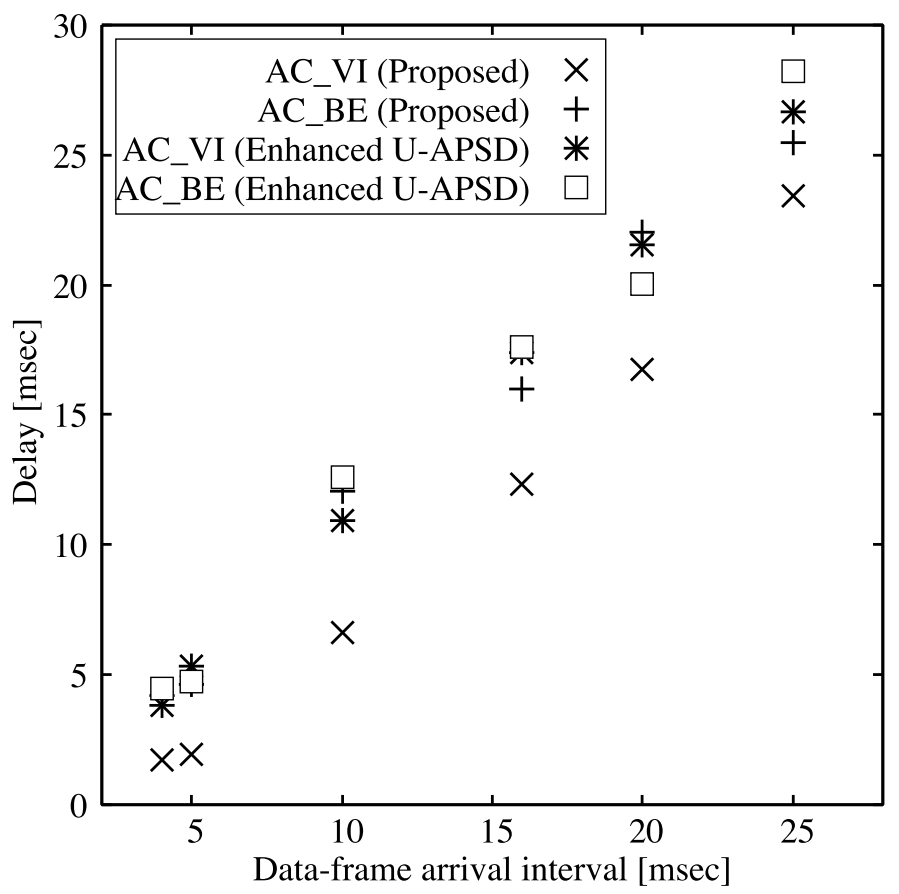

Fig. 7. Delay as a function of $T_{D L}$.

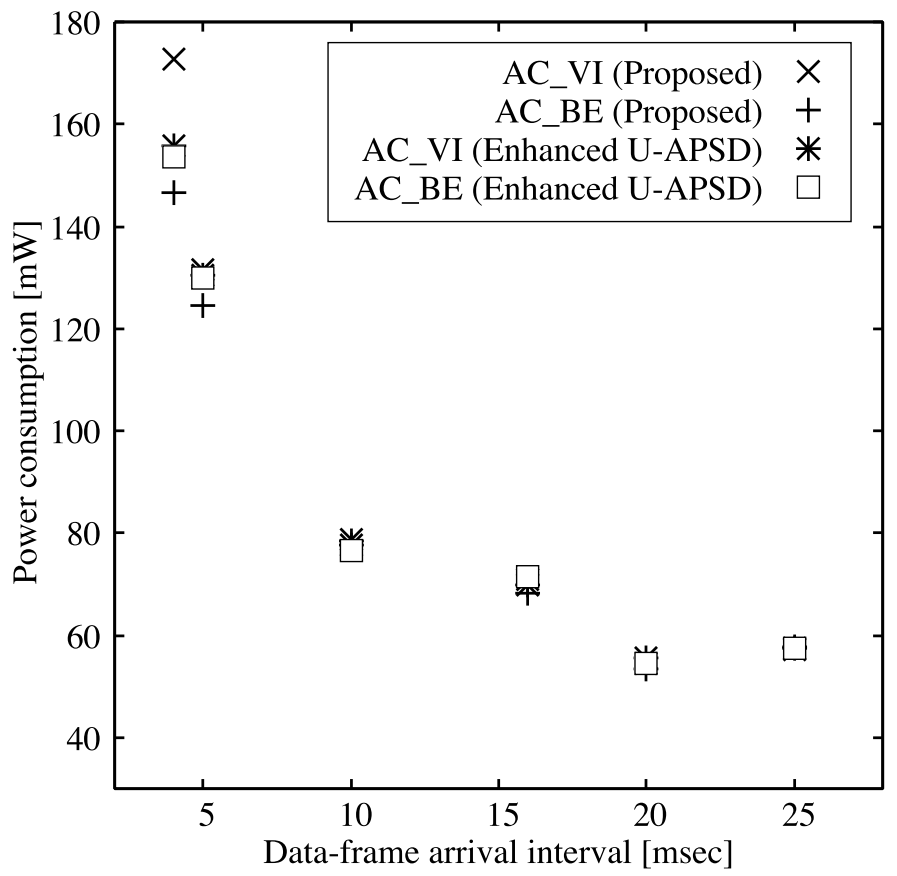

Fig. 8. Power consumption as a function of $T_{D L}$.

$T_{D L}$ is long. When $T_{D L}$ is long, the sleep period of a station is much longer than the active period, which leads to save power consumption. The power consumption of the station with AC_BE-flow of the proposed scheme is the lower than those of the other stations especially when $T_{D L}$ is short. It is seen from Figs. 7 and 8 that the proposed scheme prevented AC_VI-flow from resulting in the extra delay with saving power consumption especially when the data-frame arrival interval is short. It is also seen from Figs. 7 and 8 that that the AC_VI-flow of the proposed scheme achieves low delay with saving power when the data-frame arrival interval is long. This is because the active period becomes much shorter than the trigger interval and the sleep period as the data-frame arrival interval increases. 


\section{Conclusion}

This paper proposed a scheme for the distributed power saving mechanism of IEEE 802.11e networks in consideration of the QoS requirement. In the proposed protocol, each station enters into sleepmode and active-mode periodically according to the appropriate trigger interval. For obtaining the appropriate trigger interval, this paper presented the objective function of power consumption in consideration of the acceptable delay required by each access category. Each station optimizes its trigger interval according to the objective function. It has been seen from simulation results that the proposed scheme prevented high-priority flow from resulting in the extra delay with saving power consumption. It has also been seen from simulation results that the proposed scheme can save power consumption of the station with low-priority flow especially when the data-arrival-rate is high.

\section{References}

[1] IEEE Computer Society LAN MAN Standards Committee, "Wireless LAN MAC and PHY specifications: MAC QoS Enhancements," IEEE 802.11e Std., 2005.

[2] J. Adams et al., "Power save adaptation algorithm for multimedia streaming to mobile devices," Proc. IEEE Int'l Conf. Portable Infomation Devices, pp. 1-5, May 2007.

[3] G. Ciccarese, et al., "A novel APSD scheduler for WLAN IEEE 802.11e," Proc. IEEE/IET Int'l Symp. Communication Systems, Networks and Digital Signal Processing, pp. 19-21, July 2006.

[4] D. Camps-Mur et al., "An adaptive solution for wireless LAN distributed power saving modes," Elsevier Computer Networks, vol. 53, no. 18, pp. 3011-3030, December 2009.

[5] X. Perez-Costa et al., "IEEE 802.11e QoS and power saving features overview and analysis of combined performance," IEEE Trans. Wireless Commun., vol. 17, no. 4, pp. 88-96, August 2010.

[6] F. Wang et al., "Power-saving mechanisms for mobile devices in wireless communications," IET Commun., vol. 3, no. 2, pp. 257-267, February 2009.

[7] G.A. Safdar et al., "Improved power-saving medium-access protocol for IEEE 802.11e QoSenabled wireless networks," IET Commun., vol. 1, no. 4, pp. 718-725, August 2007.

[8] A.M. Kholaif et al., "QoS-enabled power saving access points for IEEE 802.11e networks," Proc. Wireless Communications and Networking Conference, pp. 2331-2336, April 2008.

[9] C. Cicconetti et al., "Reducing power consumption with QoS constraints in IEEE 802.16e wireless networks," IEEE Trans. Mobile Computing, vol. 9, no. 7, pp. 1006-1021, July 2010.

[10] J.-J. Huang et al., "QoS control over the power saving in 3GPP LTE advanced wireless networks," Proc. Int'l Symp. Wireless and Pervasive Computing, pp. 1-4, November 2013.

[11] X. Zhang et al., "Optimal trade-off between power saving and QoS provisioning for multicell cooperation networks," IEEE Trans. Wireless Commun., vol. 20, no. 1, pp. 90-96, February 2013.

[12] M.S. Mushtaq et al, "QoS-aware LTE downlink scheduler for VoIP with power saving," Proc. Int'l Conf. Computational Science and Engineering, pp. 243-250, December 2012.

[13] D. Qiao et al., "Smart power-saving mode for IEEE 802.11 wireless LANs," Proc. IEEE INFOCOM, vol. 3, pp. 1573-1583, March 2005.

[14] R. Krashinsky et al., "Minimizing energy for wireless web access with bounded slowdown," Proc. ACM Int'l Conf. Mobile Computing and Networking, pp. 135-148, April 2006.

[15] N. Komuro, "An adaptive sleep period control in consideration of QoS requirement for IEEE 802.11e APSD-compliant wireless LAN," Proc. Korea-Japan Joint Workshop on Complex Communication Sciences, SA-3, October 2013. 